\title{
Le cyclone : un nouveau réacteur chimique solaire gaz-solide Application à la mise en auvre en continu de la pyrolyse flash de sciure de bois
}

\author{
J. Lede, F. Verzaro et J. Villermaux \\ Laboratoire des Sciences du Génie Chimique, CNRS-ENSIC, 1, rue Grandville, 54042 Nancy Cedex, France
}

(Reçu le 31 août 1979, révisé le 17 décembre 1979, accepté le 18 décembre 1979)

\begin{abstract}
Résumé. - Le cyclone, bien connu comme séparateur centrifuge peut être valablement utilisé comme réacteur chimique gaz-solide. Des premières expériences faites sur un cyclone du type standard chauffé à $1323 \mathrm{~K}$ au foyer d'un four à image soulignent la bonne efficacité du transfert de chaleur entre ses parois et le gaz qui le traverse. On montre, dans un deuxième temps, que les informations recueillies à la suite de la mise en œuvre en continu de la pyrolyse flash de sciure de bois à $1143 \mathrm{~K}$ sont tout à fait en accord avec celles issues d'autres expériences effectuées dans des conditions fort différentes. Cette réaction ainsi que toute autre réaction gaz-solide endothermique pourraient être mises en œuvre à une plus large échelle dans ce nouveau type de réacteur grâce à l'utilisation de l'énergie solaire concentrée.
\end{abstract}

\begin{abstract}
Cyclones, known as centrifugal separators, can be also used as gas-solid chemical reactors. Preliminary experiments made on a cyclone of a conventional type heated at $1323 \mathrm{~K}$ at the focus of an image furnace show the good efficiency of heat transfer between the walls and the gas. We have then studied the continuous flash pyrolysis of wood sawdust at $1143 \mathrm{~K}$. The results obtained agree with others issued from experiments made in quite different conditions. Such a reaction or any other endothermic gas-solid reaction could be carried out on a large scale in such a new type of reactor by use of concentrated solar energy.
\end{abstract}

1. Position du problème et objectifs. - $1.1 \mathrm{LE}$ CYCLONE : RÉACTEUR GAZ-SOLIDE. - Dans un article récent, A. Vialaron [1] soulignait l'intérêt porté actuellement à la Chimie solaire (stockage chimique, conversion en vecteurs chimiques, préparation de produits). Le développement pratique de ces applications implique la réalisation de nouveaux types de réacteurs dans le cadre d'un génie de la réaction chimique solaire [2]. Ces réacteurs doivent en particulier permettre la mise en œuvre de réactions endothermiques à haute température et la réalisation de chocs thermiques (chauffage brutal des réactifs et/ou refroidissement très rapide des produits).

Considérons les réactions gaz-solide consommable. Les réacteurs industriels peuvent être classés en trois grandes catégories : les réacteurs à lits empilés, les réacteurs à lits fluidisés ou suspendus, les réacteurs à lits transportés. Dans cette dernière catégorie, une mention particulière peut être accordée aux réacteurs assurant une mise en rotation de l'écoulement [3]. Le dispositif le plus connu est le cyclone dont la fonction habituelle est de séparer des fines particules (solides ou liquides) contenues dans un fluide. Dans la configuration habituelle (Fig. 1) le fluide entraînant les particules pénètre tangentiellement dans la partie cylindrique et prend un mouvement de vitesse dirigé vers le bas. Les particules sont projetées par la force centrifuge sur les parois du cylindre avant de se réunir dans la partie conique du cyclone. Dans cette zone, le fluide inverse sa trajectoire et ressort de l'enceinte par sa partie supérieure. De nombreux détails concernant le fonctionnement des séparateurs centrifuges sont rassemblés dans les références $[4,5]$.

Les cyclones, couramment utilisés en tant que séparateurs dans les scieries, cimenteries, etc., sont également mis en œuvre dans certaines opérations à haute température $[6,7,8]$. Ces dernières applications suggèrent l'utilisation de ces dispositifs en tant que réacteurs chimiques $[2,9,10]$, certains travaux ayant montré leurs excellentes performances en ce qui concerne le transfert de matière [11] et de chaleur $[12,13]$, ainsi que le contact gaz-solide à des temps de séjour relativement courts.

C'est dans cet esprit qu'il nous est apparu intéressant d'étudier les possibilités d'utilisation du cyclone pour la mise en œuvre de réactions gaz-solide à haute température grâce à l'énergie solaire concentrée. De nombreuses réactions susceptibles d'avoir un impact 


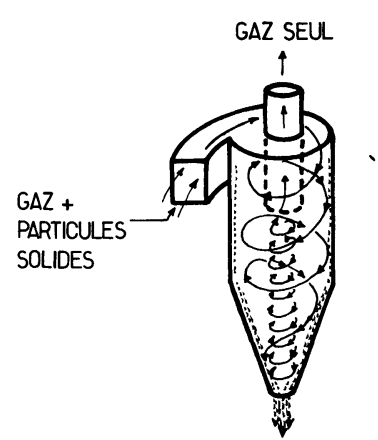

SOLIDE SEUL

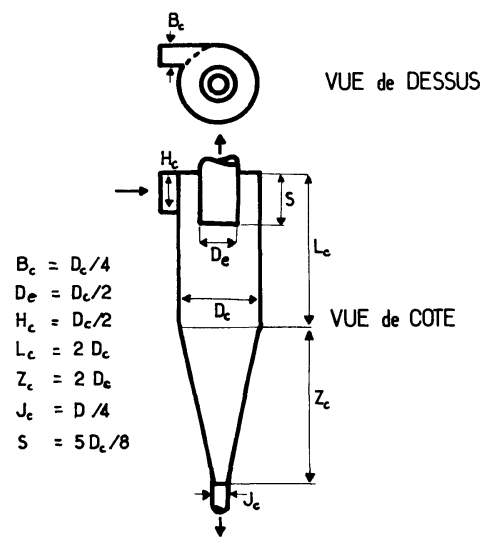

Fig. 1. - Représentation schématique d'un séparateur centrifuge (cyclone standard : trajectoires représentant l'écoulement du fluide et dimensions types)

[Schematic representation of a centrifugal separator' (conventional cyclone) : flow pattern of fluid and typical dimensions.]

industriel peuvent être envisagées dans un tel système. Nous choisirons à titre d'exemple la réaction de pyrolyse flash du bois.

1.2 LA PYROLYSE FLASH DU BOIS. - Parmi les différents procédés envisageables pour la valorisation énergétique des déchets ligno-cellulosiques, la pyrolyse flash en présence ou non de vapeur d'eau semble très intéressante. Il est possible en effet de transformer la matière végétale en un mélange gazeux riche en hydrogène, monoxyde de carbone et hydrocarbures légers de grand intérêt chimique et énergétique [14].

Le rendement en gaz de l'opération est d'autant meilleur que la température est plus élevée $(52 \%$ à $973 \mathrm{~K} ; 62 \%$ à $1273 \mathrm{~K}$ en bois sec) et que les particules solides sont portées brutalement à cette température, le passage prolongé à des températures plus basses favorisant en effet la formation de goudrons. Envisageons alors un cyclone dont les parois sont chauffées par le rayonnement solaire concentré. Les grains de bois transportés par un gaz porteur sont dès leur entrée dans le réacteur plongés au sein d'un milieu à haute température et projetés à grande vitesse sur les parois chaudes. Ils subissent ainsi une pyrolyse flash en continu. Dans le même temps, le cyclone joue son rôle de séparateur vis-à-vis des cendres qui peuvent être ainsi récupérées dans sa partie inférieure.

2. Aspect expérimental. - Les cyclones utilisés sont du type standard en ce sens que leurs dimensions relatives correspondent aux normes habituellement préconisées [4] et précisées sur la figure 1.

Trois types d'expériences ont été menées :

- Expériences de Distribution des Temps de Séjour (DTS).

- Expériences de transfert de chaleur.

- Expériences de pyrolyse flash proprement dite.

Dans le premier cas le cyclone, en altuglas, présente un diamètre de $12,5 \mathrm{~cm}$.

Dans les deux autres cas le cyclone, en acier inox, présente un diamètre de $1,68 \mathrm{~cm}$.

2.1 EXPÉRIENCES DE DTS. - Ces expériences doivent permettre, par des mesures globales, de proposer un modèle d'écoulement dans le réacteur.

De l'air circule dans le cyclone en régime permanent et à température ambiante. A un instant donné une injection impulsion d'un traceur (hydrogène) est introduite à l'aide d'une vanne à boucle à l'entrée du cyclone, dans des conditions telles que l'écoulement général ne soit pas perturbé. Un détecteur catharométrique mesure en sortie les variations de concentration de traceur. Au moyen d'un traitement sur ordinateur, on recherche un modèle d'écoulement représentatif de la courbe de réponse à l'impulsion de traceur. Ces expériences ont été effectuées à plusieurs débits. La référence [20] rassemble les détails expérimentaux relatifs à ces expériences.

2.2 EXPÉRIENCES DE TRANSFERT DE CHALEUR. Le cyclone. - Réalisé en acier inox, il présente un diamètre de $1,68 \mathrm{~cm}$, un volume de $9,64 \mathrm{~cm}^{3}$ et une surface intérieure de $32 \mathrm{~cm}^{2}$.

Le chauffage au four à image. - Afin de préparer des campagnes ultérieures au four solaire, nous avons effectué les premiers essais au four à image, qui permet de reproduire au laboratoire des conditions proches de celles régnant dans un concentrateur solaire habituel $[15,16]$.

Une lampe au xénon horizontale de $4 \mathrm{~kW}$ se trouve au foyer objet d'un miroir elliptique très enveloppant (lanterne de cinéma Cinemeccanica X $6500 \mathrm{H}$ ). Le faisceau lumineux est intercepté par un second miroir elliptique (diamètre $415 \mathrm{~mm}$ ) argenté face avant et dont le foyer objet est confondu avec le foyer image du miroir primaire. L'image de la pointe de la cathode se forme au second foyer du second miroir avec un grandissement voisin de 1 .

Dans les premiers essais, le cyclone est simplement disposé à l'emplacement du foyer. La canalisation d'entrée est refroidie par un serpentin parcouru par de l'eau froide afin d'éviter un important préchauffage du gaz et de réaliser ainsi les conditions des expé- 


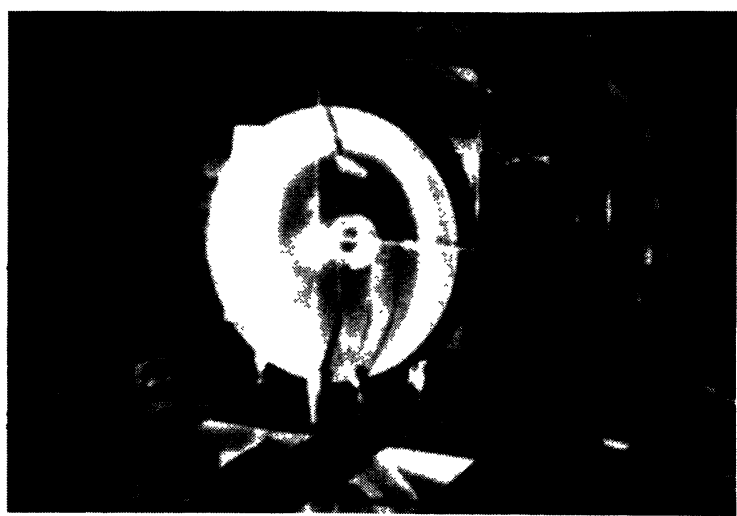

Fig. 2. -- Photographie du réacteur cyclone au foyer du four à image.

[Photograph of cyclone reactor at the focus of image furnace.]

riences de pyrolyse proprement dites dans lesquelles un choc thermique initial est requis (Fig. 2).

La température de paroi (uniforme dans la section cylindrique) est mesurée par pyrométrie à disparition de filament. La température du gaz est mesurée à l'entrée et à la sortie du cyclone (entrée du noyau central) avec un thermocouple chromel alumel.

Des expériences ont été faites avec le gaz porteur circulant seul à différents débits. Les temps de passage correspondant sont compris entre 0,1 et $0,4 \mathrm{~s}$.

2.3 EXPÉRIENCES DE PYROLYSE. - Le cyclone ainsi que le mode de chauffage sont les mêmes que ceux décrits dans la section précédente.

Alimentation en bois. - Les expériences sont menées avec de la sciure de pin Douglas dont les grains ont un diamètre compris entre 0,140 et $0,200 \mathrm{~mm}$. Ces grains sont utilisés tels quels sans humidification supplémentaire.

De nombreux procédés existent pour transporter un solide [17]. Les propriétés physiques de la sciure font que la vis sans fin serait probablement le meilleur système. Cependant pour une étude fondamentale à faible débit en solide, nous avons utilisé une technique s'inspirant d'une méthode mise au point par Dauvergne [18]. Le gaz porteur arrache par abrasion éolienne les grains situés à la surface d'un lit de sciure dont le niveau est maintenu constant par une alimentation continue et régulière (vibreur) (Fig. 3). Dans les premiers essais que nous décrivons ici, le gaz porteur est de l'argon.

Bilans de matière. - Ils sont établis à partir des pesées suivantes :

- charge de bois avant et après expérience,

- cendres récupérées à la base du cyclone,

- condensables (goudrons et eau) déposés dans la canalisation aval et dans un piège à glace.

Les gaz traversent une ampoule de $134 \mathrm{~cm}^{3}$ que l'on isole à un instant donné et dans laquelle se font

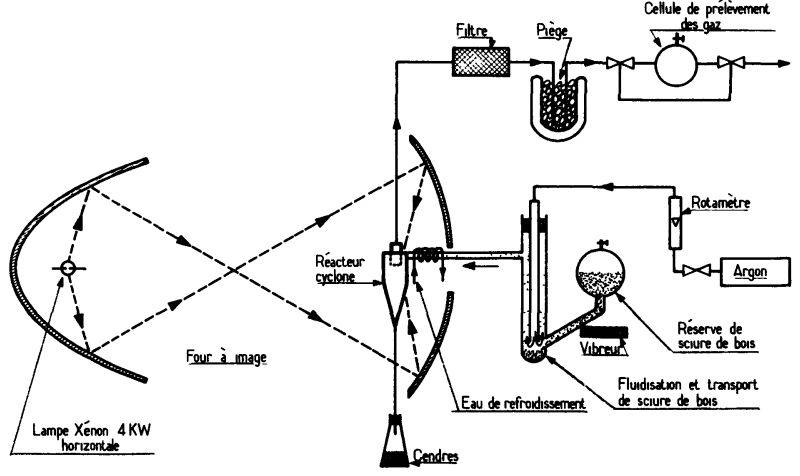

Fig. 3. - Schéma de principe de l'appareillage permettant la pyrolyse flash continue de sciure de bois dans un réacteur cyclone situé au foyer d'un four à image.

[Schematic diagram of apparatus for continuous flash pyrolysis of wood sawdust in a cyclone reactor located at the focus of an image furnace.]

les prélèvements pour analyses. Celles-ci sont effectuées par chromatographie : $\mathrm{CO}$ et $\mathrm{H}_{2}$ sont analysés sur tamis moléculaire (détection : catharomètre); $\mathrm{CH}_{4}, \mathrm{C}_{2} \mathrm{H}_{4}$ et $\mathrm{C}_{2} \mathrm{H}_{2}$ sur tamis moléculaire (détection : ionisation de flamme); $\mathrm{CO}_{2}$ sur PORAPAK Q (détection : catharomètre).

3. Résultats. - 3.1 Modèle D'éCOULEMENT. Les expériences de Distribution des Temps de Séjour permettent de proposer un modèle d'écoulement du gaz seul dans un cyclone standard. A l'intérieur du domaine exploré pour les critères de Reynolds d'entrée $\operatorname{Re}_{0}(1270$ à 2400$)$ le cyclone peut être assimilé à une zone en écoulement laminaire permanent suivie d'une zone mélangée en partie court-circuitée (Fig. 4).

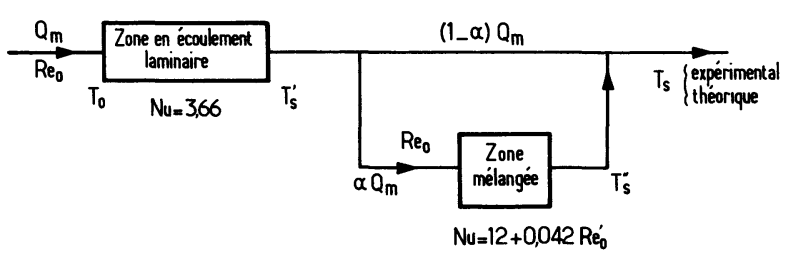

Fig. 4. - Modèle d'écoulement dans un cyclone.

[Flow pattern in a cyclone.]

La figure 5 montre que le volume $V_{1}$ de la zone laminaire varie linéairement en fonction de $R e_{0}^{2}$ selon la relation :

$$
\frac{V_{1}}{V_{\mathrm{T}}}=10^{-7} R e_{0}^{2}
$$

$V_{\mathrm{T}}$ représentant le volume total du cyclone.

La figure 6 montre que la fraction court-circuitée $1-\alpha$ varie linéairement en fonction de $R e_{0}^{3}$ :

$$
1-\alpha=3,72 \times 10^{-11} R e_{0}^{3} .
$$




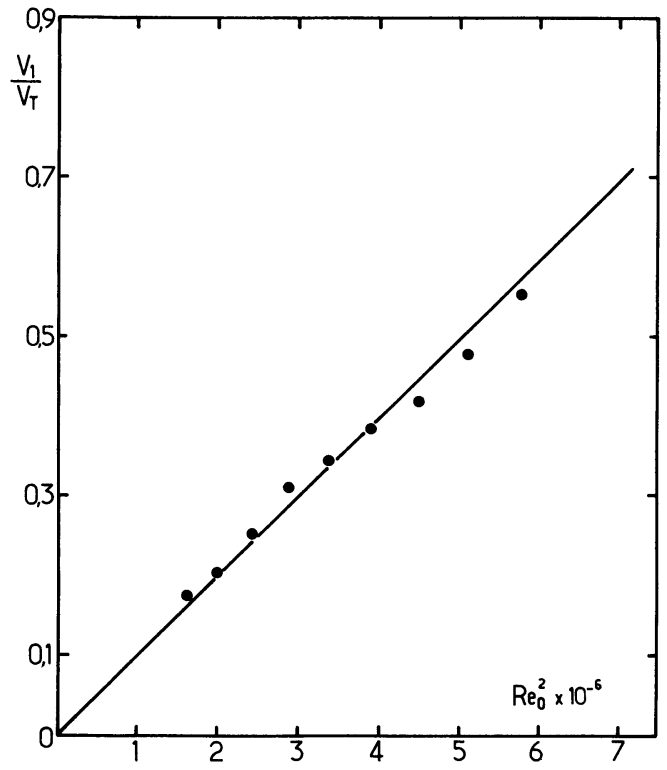

Fig. 5. - Variations du volume de la zone laminaire $\left(V_{1}\right)$ en fonction du critère de Reynolds d'entrée $\left(R e_{0}\right)$.

[Variations of laminar zone volume $\left(V_{1}\right)$ against inlet Reynolds number $\left(R e_{0}\right)$.]

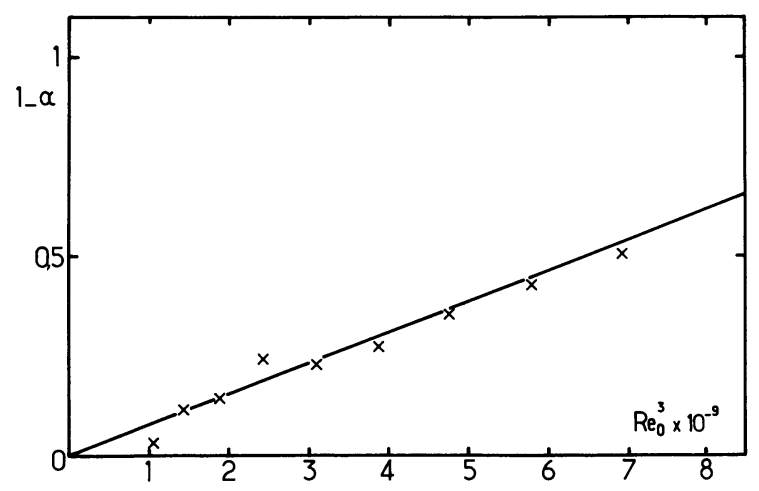

Fig. 6. - Variations de la fraction court-circuitée $(1-\alpha)$ en fonction du critère de Reynolds d'entrée $\left(R e_{0}\right)$.

[Variations of by pass fraction $(1-\alpha)$ against inlet Reynolds number.]

3.2 EFfiCACITÉ DU TRANSFERT DE CHALEUR. Remarques. - Utilisés en tant que séparateurs, les cyclones doivent être en mesure de séparer des particules très fines. A cet effet, leurs performances sont la plupart du temps estimées en étudiant l'évolution $\mathrm{du}$ diamètre de coupure (diamètre des particules collectées à $50 \%$ ) en fonction de différents paramètres (vitesse d'entrée du gaz, dimensions du cyclone, etc.). Les auteurs choisissant généralement des diamètres de coupure très petits, les vitesses d'entrée du gaz doivent être très grandes. A titre d'exemple, pour un cyclone de $15,2 \mathrm{~cm}$ de diamètre [13], une vitesse d'entrée du gaz de $15 \mathrm{~m} / \mathrm{s}$ correspond à un diamètre de coupure de $15 \mu \mathrm{m}$. L'écoulement dans la canalisation d'entrée est franchement turbulent. Dans les expériences de transfert de chaleur effectuées par Szekelly et coll. [13], le critère de Reynolds d'entrée est ainsi compris entre 20000 et 50000 .

Dans nos conditions, le diamètre des grains étant relativement plus important, il est inutile d'opérer avec de telles vitesses. Il est donc intéressant d'évaluer l'efficacité du transfert de chaleur dans ces conditions de fonctionnement moins sévères.

Résultats expérimentaux. - Ils ont été obtenus avec de l'argon circulant seul à différents débits auxquels correspondent des vitesses d'entrée légèrement supérieures à la vitesse d'entrée théorique calculable [4] à partir d'un diamètre de coupure égal à la dimension des grains de bois utilisés dans l'étude chimique.

Dans l'expérience type proposée, la température de la paroi est restée sensiblement constante (1 $323 \mathrm{~K})$ quel que soit le débit.

Les données expérimentales figurent dans le tableau I.

Dépouillement global. - Afin de comparer les performances de ce cylone avec celles déduites des expériences de Szekelly et coll. [13] nous avons calculé les critères de Reynolds $\left(R e_{0}\right)$ et de Nusselt $\left(N u_{\mathrm{sz}}\right)$ en choisissant, comme ces auteurs, le diamètre d'entrée $\left(d_{0}=0,35 \mathrm{~cm}\right)$ comme longueur caractéristique, viscosité et conductibilité thermique étant calculées aux températures d'entrée $T_{0}$ :

$R e_{0}=\frac{4 Q_{\mathrm{m}}}{\pi \eta\left(T_{0}\right) d_{0}} \quad N u_{\mathrm{sz}}=\frac{h d_{0}}{\lambda\left(T_{0}\right)}=\frac{d_{0} Q_{\mathrm{m}} C_{\mathrm{p}}\left(T_{\mathrm{s}}-T_{0}\right)}{S_{\mathrm{T}} \Delta T \lambda\left(T_{0}\right)}$.

Dans le domaine de températures explorées on utilisera les relations linéaires approchées suivantes :

$\lambda\left(T_{0}\right)=\left(0,013 T_{0}+0,35\right) \times 10^{-5} \quad\left(\mathrm{cal} . \mathrm{cm}^{-1} \mathrm{~s}^{-1} \mathrm{~K}^{-1}\right)$ $\eta\left(T_{0}\right)=\left(0,059 T_{0}+4,9\right) \times 10^{-5} \quad$ (poises) .

$\Delta T$ est une température moyenne arithmétique définie ainsi :

$$
\Delta T=\frac{\left(T_{\mathrm{p}}-T_{0}\right)+\left(T_{\mathrm{p}}-T_{\mathrm{s}}\right)}{2} .
$$

La représentation $\log _{\mathrm{e}} N u_{\mathrm{sz}}$ en fonction de $\log _{\mathrm{e}} R e_{0}$ est linéaire (Fig. 7). Un dépouillement par la méthode des moindres carrés permet de proposer la relation :

$$
N u_{\mathrm{sz}}=0,0076 R e_{0}^{0,83} .
$$

La comparaison de cette relation avec celle proposée par Szekelly et coll. $\left(N u_{\mathrm{sz}}=0,042 R e_{0}^{0,8}\right)$ montre que le transfert de chaleur est moins efficace dans nos conditions. Cette constatation pourrait s'expliquer du fait que l'écoulement à l'entrée est laminaire $\left(664 \leqslant R e_{0} \leqslant 1539\right)$ alors qu'il est franchement turbulent $\left(20000 \leqslant R e_{0} \leqslant 50000\right)$ dans les expériences de ces auteurs. 
Tableau I.

\begin{tabular}{|c|c|c|c|c|c|c|c|c|c|}
\hline No d'expérience & 1 & 2 & 3 & 4 & 5 & 6 & 7 & 8 & 9 \\
\hline$Q_{\mathrm{m}}(\mathrm{g} / \mathrm{s})$ & 0,1134 & 0,1053 & 0,0988 & 0,0907 & 0,0826 & 0,0745 & 0,0664 & 0,0583 & 0,0502 \\
\hline$R e_{0}$ & 1539 & 1424 & 1336 & 1218 & 1109 & 993 & 885 & 771 & 664 \\
\hline$N u_{\mathrm{sz}}$ & 3,37 & 3,17 & 3,01 & 2,77 & 2,59 & 2,33 & 2,11 & 1,91 & 1,69 \\
\hline$N u_{\mathrm{C}}$ & 6,9 & 6,5 & 6,2 & 5,7 & 5,3 & 4,8 & 4,4 & 3,9 & 3,5 \\
\hline$N u_{\mathrm{A}}$ & 32,71 & 31,34 & 30,06 & 27,74 & 26,64 & 24,20 & 23,26 & 21,64 & 20,04 \\
\hline$T_{0}(\mathrm{~K})$ & 372 & 373 & 374 & 377 & 377 & 380 & 381 & 384 & 384 \\
\hline$T_{\mathrm{s}}^{\prime}(\mathrm{K})$ & 550 & 566 & 582 & 594 & 574 & 572 & 583 & 586 & 616 \\
\hline$T_{\mathrm{s}}^{\prime \prime}(\mathrm{K})$ & 1089 & 1107 & 1124 & 1143 & 1157 & 1172 & 1186 & 1205 & 1227 \\
\hline$T_{\mathrm{s}}(\mathrm{th})(\mathrm{K})$ & 1015 & 1049 & 1076 & 1105 & 1128 & 1150 & 1170 & 1195 & 1220 \\
\hline$T_{\mathrm{s}}(\exp )(\mathrm{K})$ & 1058 & 1065 & 1070 & 1072 & 1083 & 1091 & 1100 & 1112 & 1125 \\
\hline $\begin{array}{c}\text { Erreur \% } \\
\frac{T_{\mathrm{s}}(\mathrm{th})-T_{\mathrm{s}}(\mathrm{exp})}{T_{\mathrm{s}}(\exp )} \times 100\end{array}$ & 4 & 1,5 & 0,6 & 3 & 4 & 5,4 & 6,4 & 7,5 & 8,4 \\
\hline
\end{tabular}

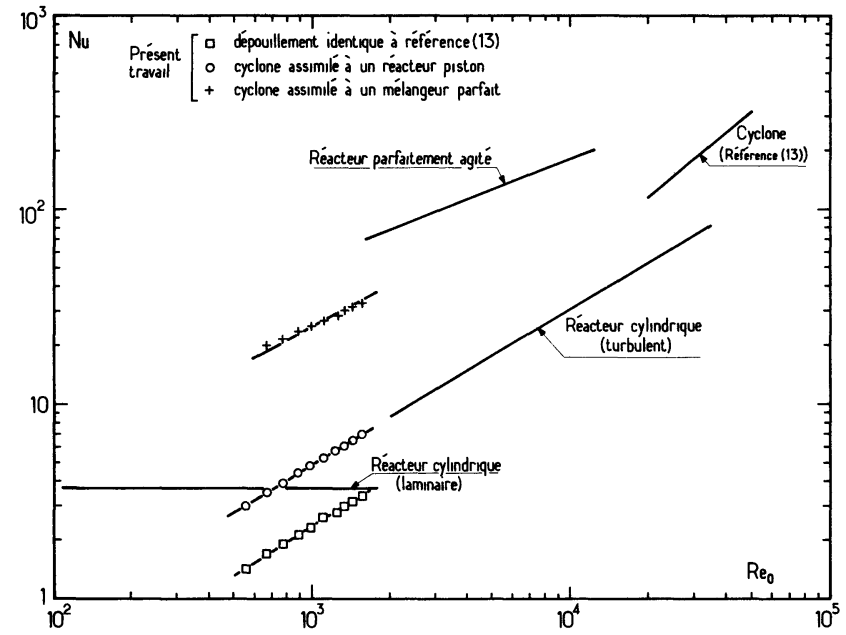

Fig. 7. - Efficacité du transfert de chaleur dans un réacteur cyclone : variations du critère de Nusselt en fonction du critère de Reynolds.

[Heat transfer efficiency in a cyclone reactor : variations of Nusselt number against Reynolds number.]

Quoi qu'il en soit, la définition du critère de Nusselt proposée nous paraît trop arbitraire. Reprenons le calcul de façon plus rigoureuse en postulant successivement deux modèles d'écoulement dans le cyclone.

- Le cyclone est assimilé à un réacteur tubulaire. Calculons le coefficient de transfert $h_{\mathrm{c}}$ correspondant à une tranche d'épaisseur $\mathrm{d} x \mathrm{du}$ cylindre équivalent (diamètre égal au diamètre hydraulique $D_{\mathrm{H}} \mathrm{du}$ cyclone). La longueur caractéristique choisie pour le calcul du nombre de Nusselt $\left(N u_{\mathrm{c}}\right)$ est $D_{\mathbf{H}}$.

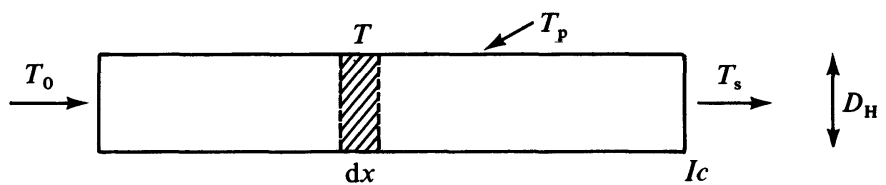

Le bilan de chaleur dans une tranche d'épaisseur $\mathrm{d} x$ s'écrit :

$$
h_{\mathrm{c}} \pi D_{\mathrm{H}}\left(T_{\mathrm{p}}-T\right) \mathrm{d} x=Q_{\mathrm{m}} C_{\mathrm{p}} \mathrm{d} T
$$

avec

$$
h_{\mathrm{c}}=\frac{N u_{\mathrm{c}} \lambda(t)}{D_{\mathrm{H}}} .
$$

Après intégration sur la longueur $l_{\mathrm{c}}$ du cylindre équivalent $\left(l_{\mathrm{c}}=S_{\mathrm{T}} / \pi D_{\mathrm{H}}\right)$ il vient :

$$
\begin{aligned}
N u_{\mathrm{c}}= & \frac{Q_{\mathrm{m}} C_{\mathrm{p}} \times 10^{5}}{\pi l_{\mathrm{c}}\left(0,013 T_{\mathrm{p}}+0,35\right)} \times \\
& \times \log _{\mathrm{e}}\left(\frac{0,013 T_{\mathrm{s}}+3,9}{T_{\mathrm{p}}-T_{\mathrm{s}}} \frac{T_{\mathrm{p}}-T_{0}}{0,013 T_{0}+0,35}\right) .
\end{aligned}
$$

Compte tenu des dimensions du cyclone, on peut calculer :

$$
\begin{aligned}
D_{\mathrm{H}} & =\frac{4 V_{\mathrm{T}}}{S_{\mathrm{T}}}=\frac{4.9,64}{31,98}=1,21 \mathrm{~cm} \\
l_{\mathrm{c}} & =8,44 \mathrm{~cm}
\end{aligned}
$$

Les valeurs de $N u_{\mathrm{c}}$ ainsi calculées figurent sur le tableau I.

En conservant la même définition du critère de Reynolds $\left(R e_{0}\right)$, la figure 7 montre que la représentation $\log _{\mathrm{e}} N u_{\mathrm{c}}$ en fonction de $\log _{\mathrm{e}} R e_{0}$ est égale- 
ment linéaire. Dans ces conditions, il est possible de proposer la relation :

$$
N u_{\mathrm{c}}=0,017 R e_{0}^{0,8 \overline{2}} .
$$

Cette relation est à rapprocher de la corrélation généralement admise pour représenter le transfert de chaleur dans un réacteur tubulaire en écoulement turbulent, à savoir :

$$
N u=0,023 \operatorname{Pr}^{0,4} \operatorname{Re}^{0,8}
$$

soit avec $\operatorname{Pr}($ argon $)=0,67$ :

$$
N u=0,020 R e^{0,8}
$$

Les relations (4) et (5) sont fort comparables. Les conditions d'écoulement dans le cyclone étant laminaires, ces résultats montrent que l'efficacité du transfert de chaleur est supérieure à celle à laquelle on pourrait s'attendre dans un réacteur tubulaire en régime laminaire $\left(N u_{\mathrm{c}}=3,66\right)$.

- Le cyclone est assimilé à un mélangeur parfait. Le bilan de chaleur s'écrit simplement :

$$
Q_{\mathrm{m}} C_{\mathrm{p}} T_{\mathrm{s}}=Q_{\mathrm{m}} C_{\mathrm{p}} T_{0}+h_{\mathrm{A}} S_{\mathrm{T}}\left(T_{\mathrm{p}}-T_{\mathrm{s}}\right) .
$$

Définissons un critère de Nusselt $N u_{\mathrm{A}}$ :

$$
N u_{\mathrm{A}}=\frac{h_{\mathrm{A}} D_{\mathrm{A}}}{\lambda\left(T_{\mathrm{s}}\right)} .
$$

$D_{\mathrm{A}}$ est le diamètre de la sphère ayant même volume [19] que le cyclone

$$
\left(D_{\mathrm{A}}=2\left(\frac{3 V_{\mathrm{T}}}{4 \pi}\right)^{1 / 3}\right)
$$

$\lambda\left(T_{\mathrm{s}}\right)$ est la conductibilité thermique du fluide calculée à la température de sortie $T_{\mathrm{s}}$ supposée uniforme dans tout le mélangeur

$$
N u_{\mathrm{A}}=\frac{Q_{\mathrm{m}} C_{\mathrm{p}} D_{\mathrm{A}}}{S_{\mathrm{T}} \lambda\left(T_{\mathrm{s}}\right)} \frac{T_{\mathrm{s}}-T_{0}}{T_{\mathrm{p}}-T_{\mathrm{s}}}
$$

Dans notre cas particulier $D_{\mathrm{A}}=2,64 \mathrm{~cm}$.

Les valeurs de $\lambda\left(T_{\mathrm{s}}\right)$ peuvent être calculées avec une bonne approximation pour $1023<T_{\mathrm{s}}(\mathrm{K})<1323$ grâce à la relation :

$\lambda=\left(0,0062 T_{\mathrm{s}}+4,31\right) 10^{-5} \quad\left(\mathrm{cal} \mathrm{cm} \mathrm{cm}^{-1} \mathrm{~s}^{-1} \mathrm{~K}^{-1}\right)$.

Les valeurs de $N u_{\mathrm{A}}$ ainsi calculées figurent dans le tableau I. La figure 7 permet d'établir la relation :

$$
N u_{\mathrm{A}}=0,17 R e_{0}^{0,72} .
$$

David et coll. (19) ont récemment proposé une corrélation permettant de prévoir l'efficacité du transfert de chaleur dans un réacteur auto-agité par quatre jets gazeux issus de quatre tuyères :

$$
N u=12+0,046 \operatorname{Pr}^{-1,5} \operatorname{Re}_{\mathrm{t}}
$$

ou avec

$$
\operatorname{Pr}=0,67: N u=12+0,084 R e_{\mathrm{t}} .
$$

$R e_{\mathrm{t}}$ est le critère de Reynolds calculé à la sortie d'une tuyère.

Assimilons le cyclone à un réacteur parfaitement agité par une seule tuyère (la canalisation d'entrée). $\mathrm{Si}$, toutes autres choses restant identiques, on égale l'énergie introduite dans le cyclone et celle introduite dans un réacteur à quatre tuyères, on montre que :

$$
R e_{0}=2 R e_{\mathrm{t}},
$$

(7) s'écrit alors : $N u=12+0,042 R e_{0}$.

Les valeurs ainsi calculées de $N u$ sont supérieures à celles correspondant à nos expériences.

La figure 7 reproduit graphiquement ces résultats et montre que le transfert de chaleur dans un cyclone est, dans nos conditions, supérieur à celui auquel on peut s'attendre dans un réacteur tubulaire, en régime laminaire et inférieur à celui correspondant à un réacteur parfaitement agité. Ces observations vont dans le sens des résultats acquis à l'issue des expériences de DTS, indiquant l'existence d'une zone laminaire suivie d'une zone mélangée.

Dépouillement basé sur les expériences de DTS. On peut utiliser les résultats des expériences de DTS et calculer pour chaque point expérimental une valeur théorique de la température de sortie en faisant appel à des valeurs ou corrélations connues pour représenter le transfert de chaleur dans la zone laminaire et dans la zone mélangée. A cet effet, assimilons la zone laminaire à un réacteur tubulaire dont le diamètre $D_{1}$ est égal au diamètre hydraulique

$$
\left(D_{1}=4 \frac{V_{1}}{S_{1}}\right) \text {. }
$$

Le gaz traverse cette zone avec une température d'entrée $T_{0}$, avec un débit massique $Q_{\mathrm{m}}$ et en ressort à une température $T_{\mathrm{s}}^{\prime}$. Une fraction $\alpha$ du débit total traverse la zone mélangée et en ressort à une température $T_{\mathrm{s}}^{\prime \prime}$. La température de sortie $T_{\mathrm{s}}(\mathrm{th})$ est celle résultant du mélange d'un débit $\alpha Q_{\mathrm{m}}$ de gaz à $T_{\mathrm{s}}^{\prime \prime}$ avec un débit $(1-\alpha) Q_{\mathrm{m}}$ de gaz à $T_{\mathrm{s}}^{\prime}$. Il pourra être intéressant de comparer $T_{\mathrm{s}}(\mathrm{th})$ aux valeurs expérimentales.

Calcul de $T_{\mathrm{s}}^{\prime}$. - Désignant par $k_{1}$ le coefficient de transfert, le bilan de chaleur dans une tranche d'épaisseur $\mathrm{d} x$ du cylindre équivalent s'écrit :

$$
h_{1} \pi D_{1}\left(T_{\mathrm{p}}-T\right) \mathrm{d} x=Q_{\mathrm{m}} C_{\mathrm{p}} \mathrm{d} T .
$$

$D_{1}$, diamètre hydraulique, peut se calculer pour chaque valeur de $Q_{\mathrm{m}}$ à partir de la valeur de $V_{1}$ correspondante (1).

En posant

$$
h_{1}=\frac{N u_{1} S_{1} \lambda(T)}{4 V_{1}}
$$

il vient :

$$
\frac{\pi N u_{1}}{Q_{\mathrm{m}} C_{\mathrm{p}}} \mathrm{d} x=\frac{\mathrm{d} T}{\left(T_{\mathrm{p}}-T\right) \lambda(T)} .
$$


Pour $550<T(\mathrm{~K})<680, \lambda(T)$ peut être obtenu avec une bonne approximation à partir de la relation : $\lambda=(0,0091 \mathrm{~T}+1,88) 10^{-5} \quad\left(\mathrm{cal} \mathrm{cm} \mathrm{cm}^{-1} \mathrm{~s}^{-1} \mathrm{~K}^{-1}\right)$.

Après intégration et avec

$$
T_{\mathrm{p}}=1323 \mathrm{~K} ; C_{\mathrm{p}}=0,124 \mathrm{cal} / \mathrm{gd}^{0} \text { (argon); }
$$

$N u=3,66$ on peut calculer $T_{\mathrm{s}}^{\prime}$ connaissant $Q_{\mathrm{m}}$ et $T_{0}$ grâce à la relation :

$$
\frac{T_{\mathrm{s}}^{\prime}+206,6}{1323-T_{\mathrm{s}}^{\prime}}=\frac{T_{0}+206,6}{1323-T_{0}} \exp \left[1,3 \times 10^{-2} \frac{l_{1}}{Q_{\mathrm{m}}}\right]
$$

$l_{1}$ étant la longueur du cylindre équivalent

$$
\left(l_{1}=4 V_{1} / \pi D_{1}^{2}\right) .
$$

Calcul de $T_{\mathrm{s}}^{\prime \prime}$. - Désignant par $h_{2}$ le coefficient de transfert, le bilan de chaleur en zone mélangée s'écrit :

$$
h_{2}=\frac{\alpha Q_{\mathrm{m}} C_{\mathrm{p}}}{S_{2}} \frac{T_{\mathrm{s}}^{\prime \prime}-T_{\mathrm{s}}^{\prime}}{T_{\mathrm{p}}-T_{\mathrm{s}}^{\prime \prime}}
$$

Posons :

$$
N u_{2}=\frac{h_{2} D_{2}}{\lambda\left(T_{\mathrm{s}}^{\prime \prime}\right)} .
$$

Nous calculerons $\mathrm{Nu}_{2}$ en utilisant la relation proposée par David et coll. [19] et permettant de prévoir l'efficacité du transfert de chaleur dans un réacteur auto-agité par jets gazeux (7). Nous assimilerons la zone mélangée à un réacteur agité par une seule tuyère ayant pour diamètre le diamètre hydraulique de la zone cylindrique laminaire. Il vient alors :

$$
N u_{2}=12+0,084 \frac{R e_{0}^{\prime}}{2}
$$

$R e_{0}^{\prime}$ est le critère de Reynolds défini sur le diamètre $D_{1}$, pour un débit $\alpha Q_{\mathrm{m}}$ et une température $T_{\mathrm{s}}^{\prime}$. Comme ces auteurs, nous prendrons $D_{2}$ égal au diamètre de la sphère ayant même volume que la zone agitée :

$$
D_{2}=\left(\frac{6 V_{2}}{\pi}\right)^{1 / 3}=\left(\frac{6\left(V_{\mathrm{T}}-V_{1}\right)}{\pi}\right)^{1 / 3} .
$$

Les valeurs de la conductibilité thermique et de la viscosité de l'argon seront calculées à partir des relations :

$1023<T(\mathrm{~K})<1323$ :

$\lambda=(0,0062 \mathrm{~T}+4,31) 10^{-5}\left(\mathrm{cal} \mathrm{cm}^{-1} \mathrm{~s}^{-1} \mathrm{~K}^{-1}\right)$,

$550<T(\mathrm{~K})<680$ :

$\eta=(0,0059 \mathrm{~T}+21) 10^{-5} \quad$ (poises)

$\alpha$ pouvant être calculé pour chaque valeur de $Q_{\mathrm{m}}(2)$, il est possible de calculer $T_{\mathrm{s}}^{\prime \prime}$, pour chaque point expérimental grâce à la relation suivante :

$$
\frac{T_{\mathrm{s}}^{\prime \prime}-T_{\mathrm{s}}^{\prime}}{1323-T_{\mathrm{s}}^{\prime \prime}}=\frac{S_{2} N u_{2}}{D_{2} \alpha Q_{\mathrm{m}}} \times 5 \times 10^{-7}\left(T_{\mathrm{s}}^{\prime \prime}+695,2\right) \text {. }
$$

Calcul de $T_{\mathrm{s}}(\mathrm{th})$. - La température $T_{\mathrm{s}}(\mathrm{th})$ des gaz en sortie du cyclone est la température de mélange ainsi définie :

$$
T_{\mathrm{s}}(\mathrm{th})=\alpha T_{\mathrm{s}}^{\prime \prime}+(1-\alpha) T_{\mathrm{s}}^{\prime} .
$$

\begin{tabular}{|c|c|c|c|c|c|}
\hline $\mathrm{Gaz}$ & $\mathrm{H}_{2}$ & $\mathrm{CO}$ & $\mathrm{CH}_{4}$ & $\mathrm{C}_{2} \mathrm{H}_{4}$ & $\mathrm{C}_{2} \mathrm{H}_{2}$ \\
\hline $\begin{array}{l}\text { Pression par- } \\
\text { tielle (torr) }\end{array}$ & 11,5 & 21,8 & 3,9 & 1,55 & 0,17 \\
\hline$\%$ molaire & 26,1 & 49,6 & 8,9 & 3,5 & 0,4 \\
\hline
\end{tabular}

Résultats. - Les valeurs de $T_{\mathrm{s}}^{\prime}, T_{\mathrm{s}}^{\prime \prime}$ et $T_{\mathrm{s}}(\mathrm{th})$ figurent dans le tableau I.

3.3 Résultats OBTENUS SUR LE PLAN CHIMIQUE. Le tableau II donne la composition des gaz recueillis pour une expérience type effectuée durant $10 \mathrm{~min}$ avec un débit d'argon de $0,039 \mathrm{~g} / \mathrm{s}$ et un débit de sciure de bois de $0,0022 \mathrm{~g} / \mathrm{s}$.

Tableau II.

Le volume de gaz dégagé est égal à $0,6 \mathrm{Nl}$ par gramme de bois et le taux de gazéification $X$ vaut :

$$
\begin{aligned}
& X=\frac{\text { Masse de gaz dégagé }}{\text { Masse de bois décomposé }}=\frac{0,758}{1,3}=0,583 \\
& X=58,3 \% .
\end{aligned}
$$

La masse de gaz dégagé $(0,758 \mathrm{~g})$ est calculée à partir de la composition.

Il a été possible de récupérer à la fin de l'expérience : $0,16 \mathrm{~g}$ de cendres et $0,196 \mathrm{~g}$ de matières condensables.

Fines particules de charbon non séparées, aérosols, etc. sont responsables des $0,186 \mathrm{~g}$ qui ont échappé aux mesures.

4. Discussion. - Les résultats rapportés dans cet article correspondent à des premiers essais de laboratoire destinés à démontrer la faisabilité du procédé proposé.

- Pour une température de paroi de $1323 \mathrm{~K}$, d'entrée de $388 \mathrm{~K}$ et un débit de $0,039 \mathrm{~g} / \mathrm{s}$, les expériences de transfert de chaleur indiquent par extrapolation une température de gaz de $1143 \mathrm{~K}$. La présence du solide ne modifie pas sensiblement cette valeur étant donné le faible débit de sciure utilisé [13]. Des expériences de pyrolyse flash effectuées en discontinu en faisant tomber de la sciure dans un four à $1173 \mathrm{~K}$ ont donné les résultats suivants [14] :

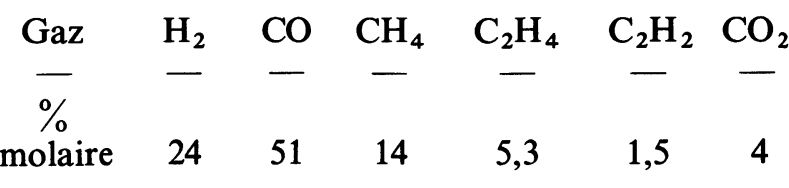

Taux de gazéification : $62,5 \%$.

Volume de gaz : 0,6 Nl/g de bois. 
On constate un bon accord entre ces valeurs et celles obtenues dans le cyclone, si ce n'est une quantité de $\mathrm{CO}_{2}$ plus importante et de $\mathrm{C}_{2} \mathrm{H}_{2}$ plus faible.

- Le dépouillement des expériences de DTS a montré que le cyclone pouvait être assimilé à un réacteur tubulaire en écoulement laminaire suivi d'un mélangeur. Cette constatation semble confirmée par les expériences de transfert de chaleur. Le tableau I indique que les valeurs de $T_{\mathrm{s}}^{\prime}$ sont relativement constantes autour d'une valeur moyenne de $580 \mathrm{~K}$. D'autre part, la confrontation des valeurs $T_{\mathrm{s}}(\exp )$ et $T_{\mathrm{s}}(\mathrm{th})$ montre un très bon accord pour les fortes valeurs de $R e_{0}$, l'écart devenant plus fort aux faibles valeurs de $R e_{0}$. Ces divergences peuvent s'expliquer du fait que le modèle d'écoulement a été déterminé pour $1270 \leqslant R e_{0} \leqslant 2400$, alors que les expériences de transfert de chaleur ont été poussées jusqu'à des valeurs beaucoup plus faibles (570); en outre, tous ces calculs théoriques ont été effectués en supposant une température de paroi constante et égale à $1323 \mathrm{~K}$, or les dimensions relatives de la tache focale et du cyclone font que la température de la base conique du cyclone (légèrement décentrée) était inférieure à $1323 \mathrm{~K}$; enfin il faut remarquer que la corrélation utilisée pour calculer $N u_{2}$ est valable pour des réacteurs parfaitement auto-agités par 4 tuyères émettant 4 jets turbulents, or nous l'avons utilisée pour une zone supposée agitée par une seule tuyère fonctionnant en régime laminaire, il est donc probable que les valeurs $\mathrm{Nu}_{2}$ calculées sont trop fortes, ce qui explique les valeurs $T_{\mathrm{s}}^{\prime \prime}$ trop élevées aux basses valeurs de $R e_{0}$.

- Le modèle proposé montre qu'il conviendrait de rechercher des géométries s'écartant légèrement des normes standards et telles que la zone laminaire (faible efficacité du transfert de chaleur), ainsi que l'importance du court-circuit soient minimisées.

- Quoi qu'il en soit le cyclone dans ses normes standards apparaît très efficace pour le chauffage d'un gaz. Pour l'expérience $n^{\circ} 1$, on peut en effet calculer une vitesse de chauffage supérieure à $8000 \mathrm{~K} / \mathrm{s}$.

Parallèlement à ces remarques, nos premières expériences suggèrent un certain nombre d'observations utiles pour une mise en œuvre future plus rationnelle du procédé.

- Des cendres ont tendance à rester accrochées sur les parois intérieures du cyclone, tendant à le colmater et à perturber l'écoulement. L'état de surface doit intervenir ici d'une manière significative. Il conviendrait d'examiner ce point lorsqu'on utilisera des débits plus importants.

- Il faut travailler à des températures plus élevées pour améliorer le taux de gazéification. Il est possible d'y parvenir en utilisant un four (solaire ou à image) plus puissant et/ou en disposant le cyclone dans une cavité absorbante vis-à-vis du rayonnement concentré. Ce dispositif est d'ailleurs indispensable si l'on veut obtenir des rendements satisfaisants. Une autre solution consisterait à faire pénétrer le rayonnement à travers le noyau central dans le cyclone jouant lui- même le rôle de cavité absorbante. Les grains seraient alors chauffés à la fois par transfert de chaleur sur les parois et par absorption directe du rayonnement. Il conviendrait dans ce cas de rechercher une disposition différente de sortie des gaz.

- Le système d'alimentation en poudre utilisé ne peut permettre que de faibles débits de solide. C'est ici un avantage car la température du gaz n'est pas sensiblement affectée par la présence du solide. Mais ce mode d'alimentation ne pourrait être employé à l'échelle productive impliquant de forts débits de solide. L'utilisation d'une vis sans fin serait probablement la meilleure solution.

- Les gaz sortent du cyclone à haute température. Il conviendrait de récupérer cette chaleur perdue afin de préchauffer les réactifs. Toutefois ce préchauffage ne doit pas dépasser $423 \mathrm{~K}$ pour éviter une décomposition prématurée du bois. Une solution consisterait à récupérer cette chaleur pour vaporiser de l'eau dont la vapeur portée vers $373 \mathrm{~K}$ constituerait le gaz vecteur. Il est en effet hors de question d'utiliser un gaz vecteur tel que l'argon dans une application pratique réelle. La vapeur d'eau serait probablement le gaz vecteur le plus indiqué, d'autant que la pyrolyse flash du bois en présence d'eau conduit à des taux de gazéification améliorés $(77 \%$ à $1273 \mathrm{~K}$ au lieu de $62 \%$ en bois sec [14]). La présence d'eau pourrait également améliorer la quantité de $\mathrm{CO}$ et $\mathrm{H}_{2}$ par réaction secondaire sur les cendres et les goudrons. La présence d'un échangeur de chaleur efficace en sortie du cyclone permettrait enfin d'éviter une décomposition des produits formés à haute température comme $\mathrm{CO}$ ou $\mathrm{C}_{2} \mathrm{H}_{2}$.

En conclusion, cette étude préliminaire montre que le réacteur cyclone est un dispositif intéressant pour la mise en œuvre de réactions gaz solide à court temps de séjour nécessitant des transferts de matière et de chaleur efficaces. Toutefois, dans un fonctionnement en tant que réacteur, la charge de solide peut être qualitativement et quantitativement différente de celle qu'on considère habituellement quand le cyclone fonctionne en séparateur. Il faut donc établir de nouvelles règles de conception et d'extrapolation. C'est notamment dans cette direction que se poursuivent les études en cours.

\section{Nomenclature}

$C_{\mathrm{p}} \quad$ Chaleur spécifique à pression constante de l'argon.

$d_{0} \quad$ Diamètre de canalisation d'entrée d'un cyclone (diamètre hydraulique pour une canalisation à section rectangulaire).

$D_{1} \quad$ Diamètre hydraulique de la zone cylindrique $\left(=4 V_{1} / S_{1}\right)$.

$D_{2} \quad$ Diamètre hydraulique de la sphère ayant même volume que la zone mélangée.

$D_{\mathrm{A}} \quad$ Diamètre de la sphère ayant même volume que le cyclone. 
$D_{\mathrm{H}} \quad$ Diamètre hydraulique $\left(=4 V_{\mathrm{T}} / S_{\mathrm{T}}\right)$.

$h \quad$ Coefficient de transfert thermique en zone cylindrique.

$h_{2} \quad$ Coefficient de transfert en zone mélangée.

$h_{\mathrm{A}} \quad$ Coefficient de transfert de chaleur (modèle global : mélangeur).

$h_{\mathrm{C}} \quad$ Coefficient de transfert de chaleur (modèle global : cylindre).

$l_{1} \quad$ Longueur du cylindre équivalent.

$l_{\mathrm{c}} \quad$ Longueur $\mathrm{du}$ cylindre équivalent (modèle global).

$N u_{1} \quad$ Critère de Nusselt en zone cylindrique.

$\mathrm{Nu}_{2} \quad$ Critère de Nusselt en zone mélangée.

$N u_{\mathrm{A}} \quad$ Critère de Nusselt (modèle : mélangeur).

$N u_{\mathrm{C}} \quad$ Critère de Nusselt (modèle : cylindre).

$N u_{\mathrm{sz}}$

$\operatorname{Pr}$

$Q_{\mathrm{m}}$

$R e$

$R e_{0}$

$R e_{0}^{\prime}$
$R e_{\mathrm{t}}$

$S_{1}$

$S_{\mathrm{T}}$

$T$

$T_{0}$

$T_{\mathrm{p}}$

$T_{\mathrm{s}}(\exp )$ Température du gaz en sortie du cyclone (expérimental).

$T_{\mathrm{s}}(\mathrm{th}) \quad$ Température du gaz en sortie du cyclone (théorique).

$T_{\mathrm{s}}^{\prime} \quad$ Température du gaz en sortie de la zone cylindrique.

$T_{\mathrm{s}}^{\prime \prime} \quad$ Température du gaz en sortie de la zone mélangée.

$V_{1} \quad$ Volume interne de la zone cylindrique.

$V_{\mathrm{T}} \quad$ Volume interne total du cyclone.

$x \quad$ Variable d'intégration.

$X \quad$ Taux de gazéification.

$\alpha \quad$ Fraction du débit passant en zone mélangée.

$\lambda(T)$ Conductibilité thermique à une température $T$.

$\eta(T) \quad$ Viscosité dynamique à une température $T$.

\section{Bibliographie}

[1] Vialaron, A., Entropie 85 (1979) 21.

[2] Villermaux, J., Entropie 85 (1979) 25.

[3] Fiche Technique GEFGN, Rev. Géné. Therm. Fr. 162 (1975) 501.

[4] Theodore, L., Buonicore, A. J., Industrial Air Pollution Control Equipment for Particulates (CRC Press Inc) 1976.

[5] Strauss, W., Industrial Gas Cleaning, 2nd Edition (Pergamon Press) 1975.

[6] Vol'f Kovich, S. I., Ionass, A. A., Mel'Nikov, E. B., Remen, R. E., Sidel'Kovskil, L. N., Troyankin, Yu. V., ShURygin, A. P., Yagodina, T. N., Int. Chem. Eng. 2 (1962) 98.

[7] REH, L., Chem. Ing. Tech. 49 (1977) 786.

[8] Encyclopedia of Chemical Processing and Design. Part 6 : Calcination Equipment to Catalysis. M. Dekker Inc. (1978).

[9] JACOBS, J., GuntheR, R., Symposium on « High Temperature Chemical Reaction Engineering ». I. Chem. E. Symposium Series No 43, Harrogate (1975).
[10] Szekelly, J., Evans, J. W., John, H. Y., Gas Solid Reactions (Academic Press) 1976.

[11] Beenackers, A. A. C. M., van SwaaiJ, N. P. M., Proc. Eur. Chem. Eng. Symp. VI 260-270, Heidelberg (1976).

[12] Klucovsky, P., Haspra, J., Duky,, J., Int. Chem. Eng. 2 (1962) 279

[13] Szekelly, J., Carr, R., Chem. Eng. Sci. 21 (1966) 1119.

[14] Lede, J., Berthellot, P., Villermaux, J., Rolin, A., FranÇOIS, H., Deglise, X., Revue Phys. Appl. Numéro spécial Energie Solaire.

[15] Foex, M., Journal des Recherches du CNRS 65 (1963) 631.

[16] Chaudron, G., Trombe, F., Les Hautes Températures et leurs utilisations en physique et en chimie (Masson) 1973, Tome I, 276-318.

[17] Chem. Eng. Deskbook. Materials Handling. Oct. (1978).

[18] Dauvergne, J. P., Thèse Nancy (1965).

[19] David, R., Houzelot, J. L., Villermaux, J., IIIrd Eur. Conf. on Mixing. York (UK) (1979).

[20] Verzaro, F., Rapport Interne. LSGC ENSIC Nancy (1979). 